\title{
Abrupt Discontinuation of Long-term Opioid Therapy Among Medicare Beneficiaries, 2012-2017
}

\author{
Hannah T. Neprash, $P h D^{7}$, Marema Gaye, $M A^{2}$, and Michael L. Barnett, $M D \mathrm{MS}^{2,3}$ (D) \\ 'Division of Health Policy \& Management, School of Public Health, University of Minnesota, Minneapolis, MN, USA; '2Department of Health Policy and \\ Management, Harvard T. H. Chan School of Public Health, Boston, MA, USA; ${ }^{3}$ Department of Medicine, Brigham and Women's Hospital, Boston, \\ MA, USA.
}

BACKGROUND: With mounting pressure to reduce opioid use, concerns exist about abrupt withdrawal of treatment for the millions of Americans using long-term opioid therapy (LTOT). However, little is known about how patients are tapered from LTOT nationally.

OBJECTIVE: Measure national patterns of LTOT discontinuation and adherence to recommended tapering speed. DESIGN: Observational study of Medicare Part D from 2012 to 2017.

PARTICIPANTS: Using claims for a $20 \%$ sample of Medicare beneficiaries, we included patients on LTOT for 1 year or more, defined as those with $\geq 4$ consecutive quarters with $>60$ days of opioids supplied in each quarter.

MAIN MEASURES: Our primary outcome was discontinuation of LTOT, defined as at least 60 consecutive days without opioids supplied. We additionally examined whether discontinuation of LTOT was "tapered" or “abrupt" by comparing LTOT users' daily MME dose in the last month of therapy to their average daily dose in a baseline period of 7 to 12 months before discontinuation. By the last month of therapy, patients with "abrupt" discontinuation had a $<50 \%$ reduction in their average daily dose at baseline.

KEY RESULTS: From 2012 to 2017, there were 258,988 LTOT users, 17,617 of whom discontinued therapy. Adjusted rates of LTOT discontinuation increased from $5.7 \%$ of users in 2012 to $8.5 \%$ in 2017 , a $49 \%$ relative increase $(p<0.001)$. There was a similar increase in annual discontinuation rate for LTOT users on lower (26-90 MME, 5.8\% to $8.7 \%, p<0.001$ ) vs. higher doses (>90 MME, $5.3 \%$ to $7.7 \%, p<0.001$ ). The majority of LTOT discontinuations were stopped abruptly, and increased over time $(70.1 \%$ to $81.2 \%$, 2012-2017, $p<0.001$ ).

CONCLUSIONS: Medicare beneficiaries on LTOT were increasingly likely to have their therapy discontinued from 2012 to 2017 . The vast majority of discontinuing users, even those on high doses, had less than 50\% reduction in dose, which is inconsistent with existing guidelines.

KEY WORDS: opioids; long-term opioid therapy; discontinuation; Medicare.

Received May 23, 2020

Accepted December 3, 2020

Published online January 29, 2021
J Gen Intern Med 36(6):1576-83

DOI: $10.1007 / \mathrm{s} 11606-020-06402-\mathrm{Z}$

(C) Society of General Internal Medicine 2021

\section{INTRODUCTION}

In the wake of the opioid overdose crisis, hundreds of state and federal policies have been passed that restrict opioid prescribing. ${ }^{1}$ These policy changes have coincided with a $40 \%$ decrease in opioid prescribing volume since 2012, which many consider evidence of success in reducing overprescribing. ${ }^{2,3}$ However, even as the total volume of opioid prescriptions falls, millions of Americans still use opioids for prolonged periods. From 2006 to 2017, while total prescribing fell, there was a $38 \%$ increase in opioid prescriptions of 30 days or more in a national all-payer database. ${ }^{2}$ Among older adults, as many as $7 \%$ of Medicare Advantage enrollees nationally had more than 90 days of opioids prescribed annually, with a similar prevalence among patients in traditional fee-for-service Medicare. $^{4,5}$

The fate of patients on long-term opioid therapy (LTOT) as the medical community responds to years of overprescribing is not clear. Media reports and patient advocates have raised concerns that increased fear of opioid prescribing could have significant harm for millions of patients in the USA who remain on LTOT, including untreated or undertreated pain, forced opioid withdrawal, and exacerbations of mental illness leading to suicide in some reports. ${ }^{6-11}$ There is observational evidence that LTOT patients in the Veterans Administration have an elevated risk of suicide or overdose associated with discontinuation. ${ }^{12}$ Though LTOT is increasingly viewed as poorly effective for chronic non-cancer pain, it is still widely used and difficult to discontinue given the physiologic dependence that develops with opioid use. ${ }^{13}$

Little evidence exists on population-level patterns of discontinuation of LTOT, ${ }^{14}$ but policy is still moving forward in response to accumulating concerns about patient harm. Over concerns that the Centers for Disease Control (CDC) 2016 Guideline for Prescribing Opioids for Chronic Pain was leading physicians to indiscriminately curtail LTOT for patients, the CDC issued a clarification about the need to apply clinically appropriate tapers for patients on LTOT who are no longer benefitting from opioids. ${ }^{15,16}$ Following this, the US 
Department of Health and Human Services also issued guidelines on reduction and discontinuation for LTOT in 2019. ${ }^{17}$ These guidelines encourage slow LTOT tapers to minimize withdrawal symptoms, and describe a decrease of $10 \%$ per week as a "faster taper," with more rapid tapers only suggested if needed for patient safety. ${ }^{18}$ Beyond these analyses, there is little additional data on clinicians' adherence to tapering guidelines when LTOT patients discontinue therapy.

To address this evidence gap, we assessed discontinuation of LTOT nationally among traditional Medicare and Medicare Advantage beneficiaries from 2012 to 2017. We estimated whether rates of discontinuation have changed over time and the rate of tapering among patients with discontinued therapy. Understanding the patterns of opioid discontinuation can inform the active and controversial debate about how to design policy that promotes responsible opioid prescribing while protecting millions of patients on LTOT.

\section{METHODS}

\section{Data Sources}

We used the Centers for Medicare and Medicaid Services beneficiary summary, hospice, and prescription drug event files for a $20 \%$ random sample of Medicare beneficiaries from January 1, 2011, through December 31, 2017. Among the $20 \%$ sample, we then identified and limited our study sample to beneficiaries aged 18 and older with at least 24 months of continuous Medicare enrollment in parts A and B, as well as Part D prescription coverage. Because Part D prescription databases contain data for the prescriptions received by beneficiaries in Medicare Advantage Part D plans, our sample included beneficiaries continuously enrolled in either fee-forservice Medicare, Medicare Advantage, or transitioning between the two.

This study was approved by the institutional review board at Harvard Medical School, which waived the requirement for informed consent since the data were deidentified and only aggregate results would be reported.

\section{Study Population}

Our population was limited to those beginning LTOT from 2011 to 2016. We considered Medicare beneficiaries as LTOT users if they had four or more consecutive quarters (e.g., January-March and April-June) with more than 60 days of opioids supplied in each quarter. If beneficiaries fell below this threshold for 1 quarter, they were still considered to have long-term use if the following quarter had 60 days or more prescribed. We did not include beneficiaries with opioid courses shorter than 4 quarters; therefore, beneficiaries could only meet this definition beginning in 2012. To calculate days supplied of opioids, we calculated days supplied from prescriptions of butorphanol, codeine, dihydrocodeine, fentanyl, hydrocodone, hydromorphone, levorphanol, meperidine, methadone, morphine, opium, oxycodone, oxymorphone, pentazocine, propoxyphene, tapentadol, and tramadol. ${ }^{19}$ We excluded prescriptions of buprenorphine from days of opioids supplied since buprenorphine can be prescribed for treating opioid use disorder.

We identified each beneficiary's initial 12-month LTOT episode and a 12-month follow-up period and grouped beneficiaries into annual cohorts beginning in 2012 for measuring discontinuation outcomes. For example, the 2012 cohort comprised LTOT users who initiated their opioid therapy in 2011 with a follow-up period beginning in 2012. Given this definition, the sample size for the 2017 cohort is smaller than other years because fewer LTOT users initiated therapy early enough (i.e., in quarter 1 of 2016) to have a follow-up period in 2017.

We excluded beneficiaries whose initial episode began in the first quarter of 2011 (the first quarter of available Medicare data) or began in the first quarter of the beneficiary's Medicare enrollment because the true start of the beneficiary's initial LTOT episode is unknown. We also excluded beneficiaries who reached the end of the study period before the end of the 12-month follow-up period (i.e., if they initiated LTOT in quarter 2 of 2016 or later), as well as beneficiaries who died before the end of their 12-month follow-up period.

Our LTOT definition requiresd significantly more opioid use than the 120-day or shorter opioid supply used by other observational studies to define "chronic" opioid therapy. 4,20,21 We used this more restrictive definition to focus on those who likely used opioids daily and were therefore at higher risk for opioid withdrawal syndrome if therapy was discontinued.

We excluded beneficiaries with any claim for a cancer diagnosis besides non-melanoma skin cancer (ICD-9 and ICD-10 codes in Appendix Table 1) and any beneficiaries with a hospice claim. We also excluded any beneficiary on an average daily dose of 25 morphine milligram equivalents (MME) or less during their initial 12-month LTOT episode. This exclusion was made because individuals on $25 \mathrm{MME}$ or less could be using opioids infrequently as needed and may not take opioids as a daily, long-term therapy.

We calculated daily MME dose as a beneficiary's total MME over the relevant total number of days (e.g., calendar year, 6-month baseline period, or individual months). We used standard conversion tables to convert different opioid formulations into $\mathrm{MME}^{22}$

\section{Defining Discontinuation and Taper Speed Outcomes}

Our primary outcome was discontinuation of LTOT, which we defined as the presence of at least 60 consecutive days of no opioids supplied following any period after a continuous LTOT period of 12 months or more, with no observed resumption of opioid therapy until the end of a 
1-year follow-up period. With this criterion, beneficiaries could only meet the definition for discontinuation if their period of LTOT ended by November 1, 2017, which allowed for 60 days of follow-up observation.

Among those discontinuing LTOT, we characterized the speed of dose tapering by comparing the average daily MME dose during a baseline period of 7 to 12 months prior to discontinuation to the average daily MME dose in the last month of LTOT. Based on guidelines from the Department of Health and Human Services and the Food and Drug Administration, the fastest possible recommended taper speed is to decrease patient's daily MME by $25 \%$ every 2 weeks, or $50 \%$ per month. ${ }^{18,23}$ While this is likely too rapid for most LTOT patients, it serves as a boundary above which patients could not have a guidelinerecommended taper speed. Using this boundary, we divided LTOT patients with discontinuation into two groups, those whose last month of LTOT was $>50 \%$ of their baseline daily MME dose ("abrupt taper," by definition tapering faster than guidelines recommend) and those whose last month was $\leq 50 \%$ of their baseline dose (" $\geq$ $50 \%$ taper," potentially guideline concordant).

\section{Patient Covariates}

We collected information on patients' age, sex, race or ethnic group, state, dual eligibility for Medicaid and Medicare coverage, enrollment in Medicare Advantage, and disability status. Using the Chronic Conditions Warehouse algorithm, we also quantified the existence of any of 27 common chronic conditions (see Table 1). ${ }^{24}$ We also quantified patients' total MME of opioids supplied, average daily dose, and the total number of days supplied during the entirety of their initial 12month LTOT episode.

\section{Statistical Analysis}

We compared the demographic characteristics of Medicare beneficiaries with LTOT who did and did not discontinue therapy during our study period. Among those discontinuing, we also compared those who did and did not have an abrupt taper. We estimated the independent patient-level predictors of discontinuation or abrupt tapering using logistic regression controlling for all available patient characteristics and indicators for state, to control for time-invariant differences in policy and prescribing environment.

Table 1 Characteristics in the Initial 12-Month LTOT Episode of Beneficiaries with LTOT Use, by Discontinuation Status

\begin{tabular}{|c|c|c|c|c|c|}
\hline $\begin{array}{l}\text { Characteristics in initial 12-month } \\
\text { LTOT episode }\end{array}$ & $\begin{array}{l}\text { All LTOT users } \\
N=\mathbf{2 5 8 , 9 8 8}\end{array}$ & $\begin{array}{l}\text { Continuous LTOT } \\
\text { users } \\
N=\mathbf{2 4 1 , 3 7 1}\end{array}$ & $\begin{array}{l}\text { Discontinued users } \\
N=17,617\end{array}$ & $\begin{array}{l}\text { aOR for } \\
\text { discontinuation }\end{array}$ & $p$ value \\
\hline Age in years (mean, SD) & $60.3(12.6)$ & $\begin{array}{l}60.4 \\
(12.6)\end{array}$ & $\begin{array}{l}58.4 \\
(13.7)\end{array}$ & 0.99 & $p<0.001$ \\
\hline \multicolumn{6}{|l|}{ Gender } \\
\hline Male & $109,045(42.1 \%)$ & $100,611(41.7 \%)$ & $8434(47.9 \%)$ & Ref. & - \\
\hline Female & $149,943(57.9 \%)$ & $140,760(58.3 \%)$ & $9183(52.1 \%)$ & 0.78 & $p<0.001$ \\
\hline \multicolumn{6}{|l|}{ Race } \\
\hline Non-white & $46,666(18 \%)$ & $43,403(18 \%)$ & $3263(18.5 \%)$ & Ref. & - \\
\hline White race & $210,711(81.4 \%)$ & $196,447(81.4 \%)$ & $14,264(81 \%)$ & 1.04 & 0.100 \\
\hline \multicolumn{6}{|c|}{ Original reason for Medicare enrollment } \\
\hline Age and survivors insurance & $74,385(28.7 \%)$ & $69,916(29 \%)$ & $4469(25.4 \%)$ & Ref. & - \\
\hline Disability & $182,196(70.3 \%)$ & $169,206(70.1 \%)$ & $12,990(73.7 \%)$ & 0.90 & $p<0.001$ \\
\hline ESRD & $980(.4 \%)$ & $915(.4 \%)$ & $65(.4 \%)$ & 0.75 & 0.031 \\
\hline Both ESRD and disability & $1427(.6 \%)$ & $1334(.6 \%)$ & $93(.5 \%)$ & 0.75 & 0.009 \\
\hline \multicolumn{6}{|l|}{ Enrolled in Medicaid } \\
\hline No & $135,892(52.5 \%)$ & $127,688(52.9 \%)$ & $8204(46.6 \%)$ & Ref. & - \\
\hline Yes & $123,096(47.5 \%)$ & $113,683(47.1 \%)$ & $9413(53.4 \%)$ & 1.19 & $p<0.001$ \\
\hline \multicolumn{6}{|l|}{ Enrolled in Medicare Advantage } \\
\hline No & $145,866(56.3 \%)$ & $135,829(56.3 \%)$ & $10,037(57 \%)$ & Ref. & - \\
\hline Yes & $113,122(43.7 \%)$ & $105,542(43.7 \%)$ & $7580(43 \%)$ & 1.01 & 0.504 \\
\hline Chronic condition count (mean, SD) & $\begin{array}{l}2.9 \\
(2.9)\end{array}$ & $\begin{array}{l}2.9 \\
(2.9)\end{array}$ & $\begin{array}{l}2.8 \\
(2.9)\end{array}$ & 0.99 & 0.041 \\
\hline \multicolumn{6}{|l|}{ Distribution of avg. daily MME } \\
\hline $26-50$ & $135,521(52.3 \%)$ & $125,732(52.1 \%)$ & $9789(55.6 \%)$ & Ref. & - \\
\hline $51-100$ & $66,380(25.6 \%)$ & $62,000(25.7 \%)$ & $4380(24.9 \%)$ & 0.87 & $p<0.001$ \\
\hline $101-200$ & $35,184(13.6 \%)$ & $32,896(13.6 \%)$ & $2288(13 \%)$ & 0.83 & $p<0.001$ \\
\hline $201+$ & $21,903(8.5 \%)$ & $20,743(8.6 \%)$ & $1160(6.6 \%)$ & 0.64 & $p<0.001$ \\
\hline Median no. of total MME of opioids & 17,400 & 17,490 & $16,432.66$ & & \\
\hline$(\mathrm{IQR})$ & $(12,195-32,670)$ & $(12,232.50-32,850)$ & $(11,755-29,877.27)$ & & \\
\hline Average daily dose $>90 \mathrm{MME}$ & $64,165(24.8 \%)$ & $60,241(25 \%)$ & $3924(22.3 \%)$ & & \\
\hline Median no. of days supplied (IQR) & $\begin{array}{l}340 \\
(322-354)\end{array}$ & $\begin{array}{l}340 \\
(322-354)\end{array}$ & $\begin{array}{l}336 \\
(319-351)\end{array}$ & & \\
\hline
\end{tabular}

aOR, adjusted odds ratio: MME, morphine milligrams equivalent; IOR, interquartile range. Adjusted odds ratio estimated using multivariable logistic regression controlling for the patient characteristics in the table and fixed effects for state. The adjusted odds ratio for age is for each additional year in age, and the odds ratio for chronic condition count is for each additional chronic condition. The chronic conditions included are acquired hyperthyroidism, acute myocardial infarction, Alzheimer's disease and related disorders or senile dementia, anemia, asthma, atrial fibrillation, benign prostatic hyperplasia, cataract, chronic kidney disease, chronic obstructive pulmonary disease, diabetes, depression, heart failure, glaucoma, hip/pelvic fracture, hyperlipidemia, hypertension, ischemic heart disease, osteoporosis, rheumatoid arthritis/osteoarthritis, and stroke or transient ischemic attack. Chronic conditions related to cancer (breast cancer, colorectal cancer, prostate cancer, lung cancer, endometrial cancer) were not present because they were excluded from the study sample 
Across all beneficiaries with discontinuation, we plotted the unadjusted median and interquartile range (IQR, 25th and 75th percentiles) of daily morphine milligram equivalent (MME) dose for each patient monthly in the 6 months before discontinuation as well as the distribution of individuals' relative percent change in MME compared to a baseline average from 7 to 12 months prior to discontinuation.

For our main outcome of discontinuation, we used multivariate linear probability models to estimate adjusted rates of discontinuation annually, controlling for all available patient characteristics with robust standard errors clustered at the patient level using the margins command in Stata (v. 15). We examined these rates among pre-specific subgroups of clinically or socioeconomically vulnerable populations: those eligible for Medicare due to disability, non-white patients, Medicaid eligible patients, and those residing in rural counties. ${ }^{25}$ We also categorized adjusted rates of discontinuation into the mutually exclusive categories of abrupt taper and $\geq 50 \%$ taper. Analyses were performed in Stata (v. 15). The $95 \%$ confidence interval around reported estimates reflects 0.025 in each tail or $p \leq 0.05$.

\section{RESULTS}

We identified 258,988 LTOT users meeting our criteria, of whom $6.8 \%$ discontinued therapy from 2012 to 2017 (Appendix Fig. 1). The majority of LTOT users were eligible for Medicare due to disability $(70.3 \%$, Table 1$)$. Compared to those using LTOT continuously, beneficiaries who discontinued LTOT in the study period were more likely to be enrolled in Medicaid (47.1\% vs. $53.4 \%$, respectively, aOR $1.19, p<0.001$, Table 1$)$ and less likely to be female $(58.3 \%$ vs. $52.1 \%$, aOR $0.78, p<0.001$, Table 1$)$. Also, compared to continuous users, discontinued users were less likely to be on higher daily MME doses in their initial 12-month episode (for example, aOR 0.64, $p<0.001$ for users with 201+ MME daily). Race/ethnicity, Medicare Advantage enrollment, and chronic condition count were similar between both groups (see Table 1) (see Appendix Table 2 for a breakdown of LTOT users' state of residence by discontinuation status).

Next we examined the trajectory of LTOT dose prior to discontinuation, stratified by their average daily MME dose at baseline (Fig. 1). Overall, $72.2 \%$ of LTOT patients with discontinuation had their opioid course abruptly tapered. Regardless of their baseline dose, the median patient's average daily MME fell less than $50 \%$ prior to discontinuation, precluding the possibility of a taper within recommended guidelines. The median discontinued patient's dose fell by $0 \%, 11 \%, 21 \%$, and $31 \%$ in the last month before discontinuation for those on 50 or less, 51-100, 101-200, and 201+ daily MME at baseline, respectively (Fig. 1 and Appendix Table 3). Correspondingly, the majority of patients with discontinuation in every daily MME dose group had an abrupt taper with less than a $50 \%$ decline from baseline prior to the last month $(77 \%, 69 \%, 63 \%$, and $60 \%$ for those on 50 or less, 51-100, 101-200, and 201+ daily MME at baseline, respectively; see Appendix Table 4).

Among beneficiaries who discontinued, those with abrupt discontinuation of LTOT were older than those with a potentially guideline-concordant taper (aOR 1.01 per year of age,

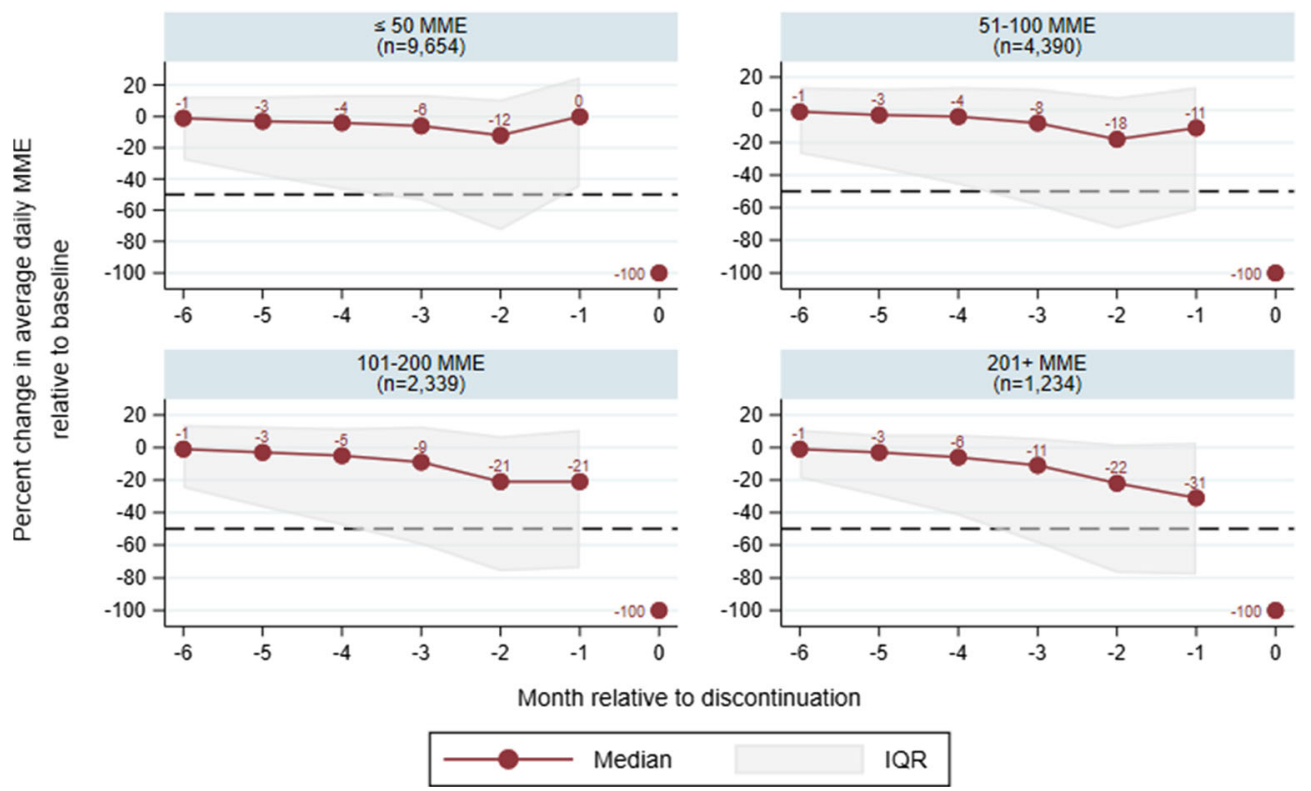

Figure 1 Relative change in daily MME dose compared to baseline period, by average daily MME at baseline (7 to 12 months before discontinuation). Each panel is labeled with the average daily MME group at baseline, and the total numbers of LTOT users in each group are in parentheses. The black dashed line demarcates a threshold of $50 \%$ of the baseline daily MME dose. Patients whose dose is above this by month -1 are classified as having an "abrupt" discontinuation, whereas those below have a " $>50 \%$ taper" that could be consistent with guidelines. While our sample excludes beneficiaries with less than 25 average daily MME in the initial 12-month LTOT episode, it is possible for beneficiaries who discontinued LTOT to have less than 25 average daily MME in the 7 to 12 months before discontinuation; therefore, the lowest dose category " $\leq 50 \mathrm{MME}$ " captures many beneficiaries with discontinuation. 
$p<0.001$, Table 2). They were also less likely to be dually eligible for Medicaid (aOR 0.82, $p<0.001$ ) and less likely to be female $(\mathrm{aOR} 0.80, p<0.001)$ than those with a potentially tapered discontinuation. Patients with higher daily MME doses were less likely to have an abrupt discontinuation (for example, aOR 0.44, $p<0.001$ for users with $201+$ MME daily). Appendix Table 5 shows a breakdown of state of residence by taper speed among users who discontinued LTOT.

From 2012 to 2017, annual adjusted rates of discontinuation increased from 5.7\% (95\% CI 5.5-5.9) of LTOT users to $8.5 \%$ (95\% CI 7.9-9.0), a 49\% relative increase (adjusted $p<0.001$, Fig. 2). Over this period, the adjusted percentage of discontinuations that were abruptly tapered increased, comprising $70.1 \%$ of discontinuations in 2012 and $81.2 \%$ in 2017 (adjusted $p<0.001)$. The relative increase in annual discontinuation rate was lower among LTOT users with higher daily MME doses in their initial 12-month episode (Appendix Table 6).

Patterns of discontinuation varied across different subgroups of potentially underserved or vulnerable patients (Table 3). LTOT users eligible for Medicare due to disability experienced a greater increase in the probability of discontinuation from 2012-2017 (adjusted rates 5.9\% to $9.2 \%, 56 \%$ relative increase) than those not eligible due to disability $(5.2 \%$ to $7.0 \%, 35 \%$ relative increase, $p<0.001$ for interaction). Individuals enrolled in Medicaid showed similar patterns of discontinuation as the Medicare-eligible-due-todisability group. Conversely, the rate of LTOT discontinuation increased less for non-white Medicare beneficiaries (adjusted rates $6.3 \%$ to $8.8 \%, 40 \%$ relative increase) than white beneficiaries $(5.6 \%$ to $8.4 \%, 50 \%$ relative increase, $p=0.02$ for interaction). Trends in the rate of LTOT discontinuation did not vary significantly by rurality.

\section{DISCUSSION}

Among Medicare Part D enrollees on long-term opioid therapy, the share experiencing discontinuation has increased in recent years. Examining opioid dose changes prior discontinuation, we also found evidence that only a minority of patients experienced a taper consistent with current guidelines. Rather, the vast majority of patients were discontinued abruptly from LTOT, falling "off a cliff" of over 50\% their daily dose at

Table 2 Characteristics in Initial 12-Month LTOT Episode of Beneficiaries Discontinuing LTOT Use, by Taper Speed

\begin{tabular}{|c|c|c|c|c|c|}
\hline $\begin{array}{l}\text { Characteristics in initial 12- } \\
\text { month LTOT episode }\end{array}$ & $\begin{array}{l}\text { Discontinued } \\
\text { users } \\
N=17,617\end{array}$ & $\begin{array}{l}\text { Tapered } \\
\text { discontinuation } \\
N=4900\end{array}$ & $\begin{array}{l}\text { Abrupt } \\
\text { discontinuation } \\
N=12,717\end{array}$ & $\begin{array}{l}\text { aOR for abrupt } \\
\text { discontinuation }\end{array}$ & $p$ value \\
\hline Age in years (SD) & $\begin{array}{l}58.4 \\
(13.7)\end{array}$ & $\begin{array}{l}56.5 \\
(13.9)\end{array}$ & $\begin{array}{l}59.1 \\
(13.6)\end{array}$ & 1.01 & $p<0.001$ \\
\hline \multicolumn{6}{|l|}{ Gender } \\
\hline Male & $8434(47.9 \%)$ & $2189(44.7 \%)$ & $6245(49.1 \%)$ & Ref. & - \\
\hline Female & $9183(52.1 \%)$ & $2711(55.3 \%)$ & $6472(50.9 \%)$ & 0.80 & $p<0.001$ \\
\hline \multicolumn{6}{|l|}{ Race } \\
\hline Non-white & $3263(18.5 \%)$ & $923(18.8 \%)$ & $2340(18.4 \%)$ & Ref. & - \\
\hline White & $14,264(81 \%)$ & $3948(80.6 \%)$ & $10,316(81.1 \%)$ & 1.00 & 0.981 \\
\hline \multicolumn{6}{|c|}{ Original reason for Medicare enrollment } \\
\hline Age and survivors insurance & $4469(25.4 \%)$ & $1080(22 \%)$ & $3389(26.6 \%)$ & Ref. & - \\
\hline Disability & $12,990(73.7 \%)$ & $3770(76.9 \%)$ & $9220(72.5 \%)$ & 1.12 & 0.039 \\
\hline ESRD & $65(.4 \%)$ & $20(.4 \%)$ & $45(.4 \%)$ & 1.05 & 0.860 \\
\hline Both ESRD and disability & $93(.5 \%)$ & $30(.6 \%)$ & $63(.5 \%)$ & 1.01 & 0.971 \\
\hline \multicolumn{6}{|l|}{ Enrolled in Medicaid } \\
\hline No & $8204(46.6 \%)$ & $2033(41.5 \%)$ & $6171(48.5 \%)$ & Ref. & - \\
\hline Yes & $9413(53.4 \%)$ & $2867(58.5 \%)$ & $6546(51.5 \%)$ & 0.82 & $p<0.001$ \\
\hline \multicolumn{6}{|l|}{ Enrolled in Medicare Advantage } \\
\hline No & $10,037(57 \%)$ & $2786(56.9 \%)$ & $7251(57 \%)$ & Ref. & - \\
\hline Yes & $7580(43 \%)$ & $2114(43.1 \%)$ & $5466(43 \%)$ & 0.79 & $p<0.001$ \\
\hline Chronic condition count (mean, & 2.8 & 2.8 & 2.7 & 0.97 & $p<0.001$ \\
\hline SD) & $(2.9)$ & $(2.9)$ & $(3.0)$ & & \\
\hline \multicolumn{6}{|l|}{ Distribution of avg. daily MME } \\
\hline $26-50$ & $9789(55.6 \%)$ & $2188(44.7 \%)$ & $7601(59.8 \%)$ & Ref. & - \\
\hline $51-100$ & $4380(24.9 \%)$ & $1393(28.4 \%)$ & $2987(23.5 \%)$ & 0.62 & $p<0.001$ \\
\hline $101-200$ & $2288(13 \%)$ & $864(17.6 \%)$ & $1424(11.2 \%)$ & 0.47 & $p<0.001$ \\
\hline $201+$ & $1160(6.6 \%)$ & $455(9.3 \%)$ & $705(5.5 \%)$ & 0.44 & $p<0.001$ \\
\hline Median no. of total MME of & $16,432.66$ & 20,400 & 15,440 & & \\
\hline opioids (IQR) & $(11,755-29,877.27)$ & $(13,189.50-38,802)$ & $(11,400-26,295)$ & & \\
\hline Average daily dose > $90 \mathrm{MME}$ & $3924(22.3 \%)$ & $1488(30.4 \%)$ & $2436(19.2 \%)$ & & \\
\hline \multirow[t]{2}{*}{ Median no. of days supplied (IQR) } & 336 & 338 & 336 & & \\
\hline & $(319-351)$ & $(321-351)$ & $(318-350)$ & & \\
\hline
\end{tabular}

aOR, adjusted odds ratio; MME, morphine milligrams equivalent; IQR, interquartile range. Adjusted odds ratio estimated using multivariable logistic regression controlling for the patient characteristics in the table and fixed effects for state. The adjusted odds ratio for age is for each additional year in age, and the odds ratio for chronic condition count is for each additional chronic condition. The chronic conditions included are acquired hyperthyroidism, acute myocardial infarction, Alzheimer's disease and related disorders or senile dementia, anemia, asthma, atrial fibrillation, benign prostatic hyperplasia, cataract, chronic kidney disease, chronic obstructive pulmonary disease, diabetes, depression, heart failure, glaucoma, hip/pelvic fracture, hyperlipidemia, hypertension, ischemic heart disease, osteoporosis, rheumatoid arthritis/osteoarthritis, and stroke or transient ischemic attack. Chronic conditions related to cancer (breast cancer, colorectal cancer, prostate cancer, lung cancer, endometrial cancer) were not present because they were excluded from the study sample 


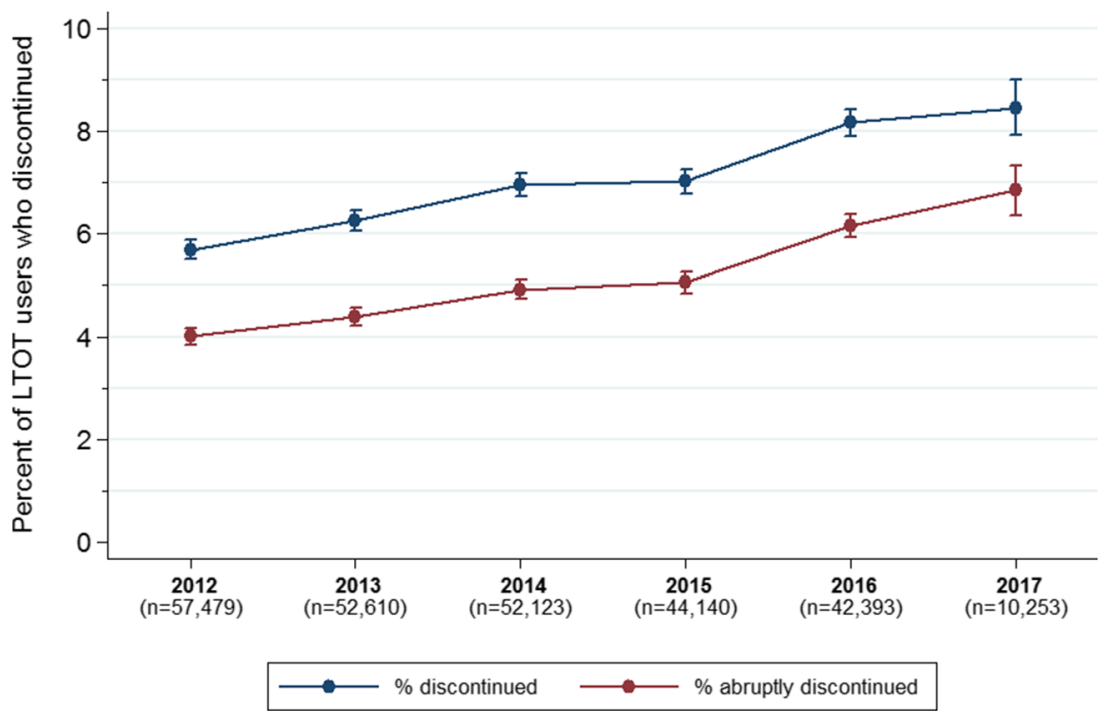

Figure 2 Trends in Adjusted Annual Rates of Discontinuation from LTOT from 2012 to 2017. For each year, the rate of discontinuation from LTOT is shown for LTOT users who initiated opioid therapy in the prior calendar year and whose 12-month follow-up period began at some point in the calendar year. The number in parentheses under each calendar year indicates the total number of LTOT users who initiated therapy in the prior year. For example, the 2012 cohort includes the 57,579 LTOT users who initiated their opioid therapy in 2011 with a follow-up period occurring some time in 2012. The sample size in 2017 is smaller because there are fewer LTOT users who initiated therapy early enough (i.e., in quarter 1 of 2016) to have a 1-year follow-up period in 2017. Rates of LTOT discontinuation were estimated using multivariate linear probability models, controlling for age, sex, race/ethnicity, state, original reason for Medicare enrollment, Medicaid enrollment, Medicare Advantage enrollment, diagnosis of chronic non-cancer pain, rurality, and total number of chronic conditions. Error bars indicate $95 \%$ CI.

baseline. While this was more common for patients on lower daily MME doses, the majority of patients on very high MME doses, even over 200 daily MME, had an abrupt discontinuation.

To our knowledge, ours is the first analysis of patterns of LTOT discontinuation among fee-for-service Medicare Part D enrollees nationally. Our findings are consistent with LTOT discontinuation trends within commercially insured patient populations ${ }^{26}$ and likely reflect the broader concurrent trend of decreasing opioid prescribing nationwide. ${ }^{2,27}$ One study of 494 Medicaid enrollees in Vermont discontinuing LTOT from 2013 to 2017 found that the vast majority of these patients had tapers faster than recommended, ${ }^{28}$ while another analysis of commercial claims data from a single insurer found that $19 \%$ of commercially insured patients were tapered beyond guideline recommendations. ${ }^{26}$ While these recent studies have found high rates of discontinuation of LTOT $^{12,29}$ and rapid tapers, ${ }^{28}$ their results are difficult to directly compare to ours because of differing definitions of the LTOT cohort and different patient populations.

Though discontinuation rates rose from 2012 to 2017, it is important to note that the vast majority of patients continued LTOT every year. Though LTOT remains an option for the management of chronic pain, it is discouraged as first-line treatment for chronic pain given its lack of superiority vs. non-opioid treatment and high rates of associated adverse effects. $^{30,31}$ The number of beneficiaries on LTOT during our study period is quite large: given that our database comprises a 20\% sample of Medicare beneficiaries, a study sample of 258,988 LTOT users implies over 1.2 million LTOT users in the entire Medicare program. The true number of beneficiaries with prolonged opioid use is almost certainly much larger because our definition of LTOT was deliberately chosen to capture an extreme level of prolonged opioid use. Therefore, while abrupt tapering of LTOT is concerning for the well-being of discontinued patients, the scope of LTOT use demonstrates the policy challenge posed by limiting opioid use while balancing the safety of LTOT users.

The nature of claims data makes it difficult to discern why we observed abrupt discontinuation so frequently. Assuming that most clinicians are aware of the severe withdrawal syndrome associated with opioid cessation, the speed of these abrupt discontinuations could be unintentional. Instead, they may reflect a lack of consensus around opioid discontinuation, guidelines for which were largely formalized after 2016. ${ }^{17,18}$ Additionally, our findings may reflect the proliferation of policy efforts at the local, state, and national level to address the opioid epidemic, including potential implicit or explicit pressure from authorities for clinicians to minimize patients' use of LTOT. While no policies that we know of directly require discontinuation of long-term opioid therapy, providers may respond to limits on prescribing for particular patient categories by reducing their opioid prescribing across the board. Finally, abrupt discontinuation of LTOT may be a consequence of a disrupted patient-provider relationship. Evidence from audit surveys suggests that providers may avoid accepting new patients on LTOT, resulting in difficulty accessing the clinical interaction that might result in a prescription. ${ }^{8}$

Regardless of the reason underlying these patterns of abrupt LTOT discontinuation, patients may be put at elevated risk for 


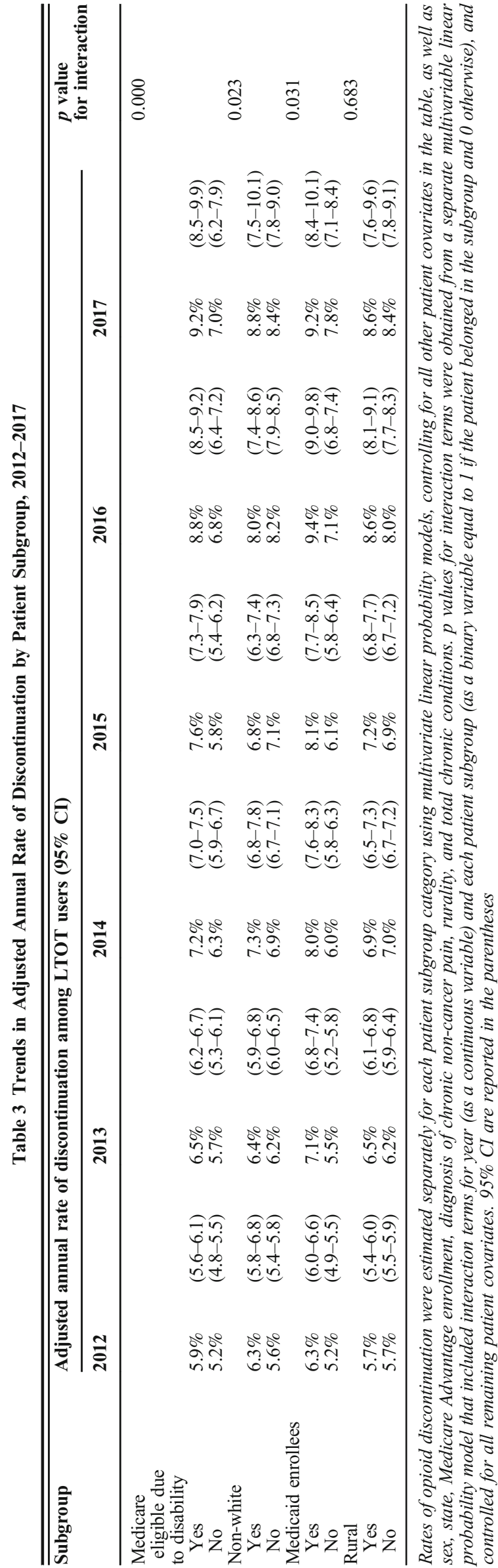

adverse health events. Limited evidence outside of the patient population studied here shows that rapidly decreasing or abruptly discontinuing LTOT significantly increases the risk of opioidrelated hospitalizations and emergency department visits. ${ }^{12,28}$

Examining the characteristics of LTOT patients who discontinued, we found a higher likelihood of discontinuation among patients dually eligible for Medicaid. This is unlikely to reflect sensitivity to the cost of prescription opioids or the office visit necessary to secure a refill since this population faces negligible cost-sharing for these services. Instead, it could reflect differences in provider perception of pain by race or socioeconomic status ${ }^{32-34}$ or policy environments in some states that target opioid use specifically within the Medicaid population. ${ }^{35}$ LTOT discontinuation did not vary meaningfully by other patient demographic characteristics, but was considerably more likely for patients on relatively low long-term doses.

There are limitations to this analysis. First, the study population is composed of Medicare beneficiaries who do not represent the general population of LTOT users; therefore, these results may not generalize to other populations. However, as the national insurer for disabled persons in the USA, LTOT use among Medicare beneficiaries has national relevance. Also, while not strictly a limitation, it is important to note that the majority of LTOT users in our sample were eligible for Medicare through disability. Therefore, our results should be viewed as predominantly reflecting LTOT use in the disabled Medicare population, who comprise the bulk of LTOT use in the program. Second, we are unable to observe the clinical appropriateness or intended taper strategy around LTOT discontinuation. Even if an opioid is prescribed for a "30-day supply" at a certain strength, it is possible that the prescriber intended the prescription to serve a tapering purpose that we are unable to observe. Therefore, our results may be biased towards abrupt discontinuation if this is a common phenomenon. Third, we used one specific definition of classifying individuals at LTOT users and another definition to differentiate abrupt vs. nonabrupt tapering. There is no consensus on the "right" definition for these concepts, so our results could differ from those using alternate definitions. Our goal in choosing definitions was to understand discontinuation among a vulnerable population of LTOT users exposed to very rapid tapering.

Our results highlight the need to monitor trends in opioid discontinuation, particularly in the wake of recent guidance issued to support clinicians as they taper and discontinue patients from opioid therapy. If this result is replicated in more recent data, physicians may need more explicit continuing medical education around guidelines for opioid therapy discontinuation and tapering. Finally, policymakers need to design policies that can discourage unnecessary opioid prescribing while protecting the health and wellbeing of LTOT users, such as monitoring opioid prescribing and deprescribing quality measures. 
Corresponding Author: Michael L. Barnett, MD MS; Department of Health Policy and Management, Harvard T. H. Chan School of Public Health, Boston, MA, USA (e-mail: mbarnett@hsph.harvard.edu).

Supplementary Information The online version contains supplementary material available at https://doi.org/10.1007/s11606-020 06402-z.

Funding Supported by grants from the RRF Foundation for Aging and the National Institute on Aging (K23 AG058806-01, MLB).

\section{Compliance with Ethical Standards:}

Conflict of Interest: Dr. Barnett is retained as an expert witness for government plaintiffs in lawsuits against opioid manufacturers. The other authors have no other conflicts of interest to disclose.

\section{REFERENCES}

1. Injury Prevention Legislation Database | Opioid Abuse Prevention. (Accessed May 25, 2018 at http://www.ncsl.org/research/health/injury-prevention-legislation-database.aspx\#monday)

2. Schieber LZ, Guy GP, Seth P, et al. Trends and patterns of geographic variation in opioid prescribing practices by state, United States, 20062017. JAMA Netw Open. 2019;2(3):e190665-e190665.

3. Zhu W, Chernew ME, Sherry TB, Maestas N. Initial opioid prescriptions among U.S. commercially insured patients, 2012-2017. N Engl J Med. 2019;380(11): 1043-52.

4. Jeffery MM, Hooten WM, Henk HJ, et al. Trends in opioid use in commercially insured and Medicare Advantage populations in 2007-16: retrospective cohort study. BMJ. 2018;362:k2833.

5. Kuo Y-F, Raji MA, Chen N-W, Hasan H, Goodwin JS. Trends in opioid prescriptions among part D medicare recipients from 2007 to 2012. Am J Med 2016;129(2):221.e21-30.

6. Hoffman J. Medicare is cracking down on opioids. Doctors fear pain patients will suffer. N. Y. Times. 2018;(Accessed Sep 18, 2018 at https:// www.nytimes.com/2018/03/27/health/opioids-medicare-limits.html)

7. Szalavitz M, Rinkunas $\mathbf{S}$. The sad cases of pain patients getting their doses cut and taking their own lives. Tonic. 2017; (Accessed Sep 18, 2018 at https://tonic.vice.com/en us/article/8x5m7g/opioid-crackdown-chronic-pain-patients-suicide)

8. Lagisetty PA, Healy N, Garpestad C, Jannausch M, Tipirneni R Bohnert ASB. Access to primary care clinics for patients with chronic pain receiving opioids. JAMA Netw Open. 2019;2(7):e196928-e196928.

9. Human Rights Watch. "Not Allowed to Be Compassionate" | Chronic Pain, the Overdose Crisis, and Unintended Harms in the US. Hum. Rights Watch. 2018;(Accessed Apr 30, 2019 at https://www.hrw.org/ report/2018/12/18/not-allowed-be-compassionate/chronic-pain-overdose-crisis-and-unintended-harms-us)

10. Bob Young. Chronic-pain patients feel sting of Washington state's opioid crackdown. 2017; (Accessed at https://www.seattletimes.com/seattlenews/health/chronic-pain-patients-feel-sting-of-washington-states-opioid-crackdown/)

11. Stone $\mathbf{W}, \mathrm{KJZZ}$. Patients with chronic pain feel caught in an opioidprescribing debate. Kais. Health News. 2018;(Accessed Aug 1, 2018 at https://khn.org/news/patients-with-chronic-pain-feel-caught-in-anopioid-prescribing-debate/)

12. Oliva EM, Bowe T, Manhapra A, et al. Associations between stopping prescriptions for opioids, length of opioid treatment, and overdose or suicide deaths in US veterans: observational evaluation. BMJ 2020;368 (Accessed Mar 5, 2020 at https://www.bmj.com/content/368/bmj. $\mathrm{m} 283)$

13. Wood E, Simel DL, Klimas J. Pain management with opioids in 20192020. JAMA. 2019;1-3.

14. Frank JW, Lovejoy TI, Becker WC, et al. Patient outcomes in dose reduction or discontinuation of long-term opioid therapy: a systematic review. Ann Intern Med. 2017:167(3):181.
15. Dowell D, Haegerich TM, Chou R. CDC guideline for prescribing opioids for chronic pain. JAMA. 2016;315(15): 1624-45.

16. Dowell D, Haegerich T, Chou R. No shortcuts to safer opioid prescribing. N Engl J Med. 2019;380(24):2285-7.

17. Dowell D, Compton WM, Giroir BP. Patient-centered reduction or discontinuation of long-term opioid analgesics: the HHS guide for clinicians. JAMA. 2019;1-3.

18. HHS guide for clinicians on the appropriate dosage reduction or discontinuation of long-term opioid analgesics. (Accessed Oct 28, 2019 at https://www.hhs.gov/opioids/sites/default/files/2019-10/Dosage_Reduction_Discontinuation.pdf)

19. Data Resources | Drug Overdose | CDC Injury Center. 2019;(Accessed Aug 18, 2020 at https://www.cdc.gov/drugoverdose/resources/data. html)

20. Sun EC, Darnall BD, Baker LC, Mackey S. Incidence of and risk factors for chronic opioid use among opioid-naive patients in the postoperative period. JAMA Intern Med. 2016;176(9):1286-93.

21. Cuthbert CA, Xu Y, Boyne DJ, Kong S, Hemmelgarn BR, Cheung WY. Patient level factors associated with chronic opioid use in cancer patients. J Clin Oncol 2019;37(15_suppl):11580-11580.

22. Center for Medicare and Medicaid Services. Opioid Oral Morphine Milligram Equivalent (MME) Conversion Factors. (Accessed Aug 13, 2018 at https://www.cms.gov/Medicare/Prescription-Drug-Coverage/ PrescriptionDrugCovContra/Downloads/Opioid-Morphine-EQ-Conversion-Factors-April-2017.pdf)

23. Research C for DE and. Drug Safety and Availability - FDA identifies harm reported from sudden discontinuation of opioid pain medicines and requires label changes to guide prescribers on gradual, individualized tapering. (Accessed Apr 17, 2019 at https://www.fda.gov/Drugs/ DrugSafety/ucm635038.htm)

24. Center for Medicare and Medicaid Services. Chronic Conditions Data Warehouse. 2014;(Accessed Mar 25, 2015 at https://www.ccwdata.org/)

25. Data Access - Urban Rural Classification Scheme for Counties. 2019;(Accessed Jun 12, 2019 at https://www.cdc.gov/nchs/data_access/urban_rural.htm)

26. Fenton JJ, Agnoli AL, Xing G, et al. Trends and rapidity of dose tapering among patients prescribed long-term opioid therapy, 20082017. JAMA Netw Open. 2019;2(11):e1916271-e1916271.

27. Medicine Use and Spending in the U.S. - IQVIA. (Accessed May 15, 2019 at https://www.iqvia.com/institute/reports/medicine-use-andspending-in-the-us-a-review-of-2018-and-outlook-to-2023)

28. Mark TL, Parish W. Opioid medication discontinuation and risk of adverse opioid-related health care events. J Subst Abuse Treat. 2019; 103:58-63.

29. James JR, Scott JM, Klein JW, et al. Mortality after discontinuation of primary care-based chronic opioid therapy for pain: a retrospective cohort study. J Gen Intern Med 2019;(Accessed Sep 6, 2019 at https:// doi.org/10.1007/s11606-019-05301-2)

30. Barnett ML. Opioid prescribing in the midst of crisis - myths and realities. N Engl J Med. 2020;382(12):1086-8.

31. Busse JW, Wang $\mathbf{L}$, Kamaleldin $\mathbf{M}$, et al. Opioids for chronic noncancer pain: a systematic review and meta-analysis. JAMA. 2018;320(23):244860.

32. Campbell CM, Edwards RR. Ethnic differences in pain and pain management. Pain Manag. 2012;2(3):219-30.

33. Johnson TJ, Weaver MD, Borrero $\mathbf{S}$, et al. Association of race and ethnicity with management of abdominal pain in the emergency department. Pediatrics. 2013;132(4):e851-8.

34. Hoffman KM, Trawalter S, Axt JR, Oliver MN. Racial bias in pain assessment and treatment recommendations, and false beliefs about biological differences between blacks and whites. Proc Natl Acad Sci U S A. $2016 ; 113(16): 4296-301$.

35. States reporting Medicaid FFS pharmacy benefit management strategies for opioids in place. Henry J Kais. Fam. Found. 2018;(Accessed Apr 3, 2020 at https://www.kff.org/medicaid/state-indicator/states-reporting-medicaid-ffspharmacy-benefit-management-strategies-for-opioids-in-place/)

Publisher's Note: Springer Nature remains neutral with regard to jurisdictional claims in published maps and institutional affiliations. 